\title{
CORRIGENDUM
}

\section{Assessment of tumor characteristics based on glycoform analysis of membrane-tethered MUC1}

Atsushi Matsuda, Michiyo Higashi, Tomomi Nakagawa, Seiya Yokoyama, Atsushi Kuno, Suguru Yonezawa and Hisashi Narimatsu

Laboratory Investigation (2017) 97, 1262; doi:10.1038/labinvest.2017.67

Correction to: Laboratory Investigation advance online publication, 5 June 2017; doi:10.1038/labinvest.2017.53
In the paper, corresponding author Dr A Kuno has only a $\mathrm{PhD}$, not an $\mathrm{MD}$. 